\title{
First-order neutron-deuteron scattering in a three-dimensional approach
}

\author{
K. Topolnicki1 ${ }^{1,2, a}$, J. Golak ${ }^{1}$, R. Skibiński ${ }^{1}$, H. Witała ${ }^{1}$, and C.A. Bertulani ${ }^{2}$ \\ 1 M. Smoluchowski Institute of Physics, Jagiellonian University, PL-30348, Kraków, Poland \\ 2 Texas A\&M University Commerce, Department of Physics and Astronomy, Commerce, Texas, USA
}

Received: 29 July 2015

Published online: 23 October 2015

(c) The Author(s) 2015. This article is published with open access at Springerlink.com

Communicated by Shi-Lin Zhu

\begin{abstract}
The description of the neutron-deuteron scattering process has been possible using the partial wave approach since the 1980s (Few-Body Syst. 3, 123 (1988); Phys. Rep. 274, 107 (1996); Acta Phys. Pol. B 28, 1677 (1997)). In recent years the so-called "three-dimensional" formalism was developed, where the calculations are performed with operators acting directly on the three-dimensional degrees of freedom of the nucleons. This approach avoids a tedious step of the classical calculations, the partial wave decomposition of operators, and in this paper is applied to the neutron-deuteron scattering process. The calculations presented here are a first step toward a new calculation scheme that would make it possible to easily produce precise predictions for a wide range of nuclear force models. This paper is a continuation of the work presented in Eur. Phys. J. A 43, 339 (2010) where the breakup channel was considered in detail. The theoretical formulation used in this paper is very closely related to the formalism introduced in Eur. Phys. J. A 43, 339 (2010) and Phys. Rev. C 68, 054003 (2003), however, we work directly with the matrix representation of operators in the joined isospin-spin space of the three-nucleon system and use only the driving term of the three-nucleon Faddeev equations. This greatly simplifies the numerical realization of the calculation and allows us to consider also the elastic channel of the reaction.
\end{abstract}

\section{Introduction}

The nucleon-deuteron $(\mathrm{Nd})$ elastic scattering is a process of great importance to study three-nucleon $(3 \mathrm{~N})$ physics. In recent years many exact theoretical formalisms have been developed to describe this process at low and intermediate energies [1-4]. In combination with continuous progress in numerical methods and computational resources this allows one to make exact predictions based both on (semi) phenomenological [5-8] forces as well as on potentials derived within the framework of chiral effective field theory $[9,10]$. It was proven $[11,12]$ that a three-nucleon force (3NF) gives a significant contribution to the elastic scattering cross section and spin observables at higher energies, which makes this process extremely interesting for studies on the $3 \mathrm{~N}$ Hamiltonian.

Also from the experimental side the proton-deuteron scattering have been extensively investigated by many groups, see for example [13-15] for recent experiments. The comparison of theoretical predictions with the data clearly shows, that $3 \mathrm{NF}$ effects grow with energy. At

\footnotetext{
a e-mail: kacper.topolnicki@uj.edu.pl
}

higher energies discrepancies between predictions and data exist. These discrepancies cannot be removed by relativistic corrections $[16,17]$ but have to originate from missing short-ranged parts of modern 3NFs. Due to the big number of partial waves required at higher energies, theoretical formalisms which are based on the partial wave decomposition (PWD) become not appropriable to describe Nd scattering at energies above around $250 \mathrm{MeV}$. Therefore it is desired to develop exact theoretical methods applicable at these energies which avoid partial wave decomposition. For a short review of different formulations avoiding PWD in the momentum space we refer to [11].

In this paper we apply the so-called "threedimensional" (3D) formalism to neutron-deuteron (nd) scattering. Our nonrelativistic description is based on the $3 \mathrm{~N}$ Faddeev equations $[1,18]$ where, instead of resorting to the PWD of operators involved in the calculations, we use the general parity and time reversal invariant form of the two-nucleon $(2 \mathrm{~N})$ potential and work with operators and states constructed from three-dimensional vectors. Our approach avoids the PWD and works directly with the operator form of the potential making the calculations easily extensible to new models of nuclear forces. In 
spite of the restriction to the leading term of the $3 \mathrm{~N}$ Faddeev equations, this work is the first step in the direction of the full $3 \mathrm{D}$ treatment of $3 \mathrm{~N}$ scattering.

The results presented in this paper are a natural continuation of the work presented in [19]. Following the formalism introduced in [19] we restrict ourselves to the driving term only but additionally extend the formalism to elastic nd scattering. This was, in part, made possible by not treating the spin and isospin spaces of the $3 \mathrm{~N}$ system separately since constructing operators, especially permutation ones, in the joined isospin-spin space is a much simpler task. It should, however, be stressed that the formalism we present here is equivalent to the one used in [19]. The expressions presented in [19] were cross-checked with the ones presented in this work. The only difference is the combined treatment of operators in the isospin and spin spaces of the $3 \mathrm{~N}$ system that greatly simplifies the calculations.

The aim of the calculations presented here is to take the first step in the direction of the full 3D treatment of three nucleon $(3 \mathrm{~N})$ scattering. We are aware that using the driving term only is insufficient to properly describe experimental results at the energies considered in this paper. Nonetheless, in sect. 4 we provide a comparison with the full PWD calculation that includes all terms. In order to verify our calculations we compare them to results obtained using well-established codes that are based on PWD, but restricted only to the driving term. In all cases we observe a very good convergence of the PWD results to our $3 \mathrm{D}$ calculations.

We use a notation in which vectors are denoted using bold face $(e . g ., \boldsymbol{p})$, unit vectors are denoted using a hat $(e . g . \hat{\boldsymbol{p}})$ and operators are denoted using an inverted hat $($ e.g. $\check{O})$. The paper is organized as follows. Section 2 contains our formalism. First, the scattering amplitudes for the breakup and elastic scattering, in their full form and with the driving term only, are introduced. The next sect. 3 describes the building blocks of the calculations. We supply the final expressions for the scattering amplitudes in terms of full $3 \mathrm{~N}$ isospin-spin operators in sect. 3.1. These expressions can serve as a good starting point in an attempt to reproduce our results. The detailed description of the formal ingredients can be found in [20-22]. In this paper we give an overview of the necessary deuteron wave function and $2 \mathrm{~N}$ transition operator calculations in subsects. 3.2 and 3.3, respectively. Next, in subsect. 3.4, we describe the permutation operators in the full isospinspin space of the $3 \mathrm{~N}$ system. Finally, in sect. 4 we present numerical results and summarize in sect. 5 .

\section{Theoretical description}

The properties of the $3 \mathrm{~N}$ system under consideration are governed by the Hamiltonian operator,

$$
\check{H}=\check{H}_{0}+\sum_{i=1}^{3} \check{V}_{i}
$$

where $\check{H}_{0}=\frac{\check{p}^{2}}{m}+\frac{3 \check{q}^{2}}{4 m}$ is the kinetic energy operator with Jacobi momenta $\boldsymbol{p}$ and $\boldsymbol{q}, m$ is the nucleon mass and $\check{V}_{i}$ is the $2 \mathrm{~N}$ potential operator for particles $l, n$ such that $i \neq l \neq n \neq i$. The key ingredients of our formalism are the two-body transition operator $\check{t}_{i}$ satisfying the LippmannSchwinger equation (LSE) [18],

$$
\check{t}_{i}(E+i \epsilon)=\check{t}_{i}(E+i \epsilon)+\check{V}_{i} \check{G}_{0}(E+i \epsilon) \check{t}_{i}(E+i \epsilon),
$$

and the deuteron bound state. In (2) the LSE is written in the $3 \mathrm{~N}$ space, $G_{0}(E+i \epsilon) \equiv\left(E+i \epsilon-\check{H}_{0}\right)^{-1}$ is the free $3 \mathrm{~N}$ propagator and $E$ is the $3 \mathrm{~N}$ energy. The deuteron bound state and the transition operator $\tilde{t}_{i}$ will be discussed in detail in sects. 3.2 and 3.3, respectively. In order to simplify the notation in the following the $i \epsilon$ term will be dropped, the limit $\epsilon \rightarrow 0^{+}$will be implied and the energy argument $E$ will be omitted.

The transition operators for nd scattering have a wellknown form $[1,19]$ both for breakup,

$$
\check{U}_{0}=(1+\check{P}) \check{T},
$$

as well as for elastic scattering,

$$
\check{U}=\check{P} \check{G}_{0}^{-1}+\check{P} \check{T},
$$

where the $\check{T}$ operator satisfies the $3 \mathrm{~N}$ Faddeev equation $[1$, 18]:

$$
\check{T}=\check{t} \check{P}+\check{t} \check{G}_{0} \check{P} \check{T} .
$$

In eqs. (3)-(5),

$$
\check{P}=\check{P}_{12} \check{P}_{23}+\check{P}_{13} \check{P}_{23}
$$

is the permutation operator $\left(\check{P}_{i j}\right.$ being an operator exchanging particles $i$ and $j$ ) and $\check{t} \equiv \check{t}_{1}$.

The operators (3) and (4) can be used to calculate transition amplitudes for the breakup and elastic channels:

$$
\begin{aligned}
A^{\mathrm{BU}} & =\left\langle\phi_{0}\left|\check{U}_{0}\right| \phi\right\rangle, \\
A^{\mathrm{EL}} & =\left\langle\phi^{\prime}|\check{U}| \phi\right\rangle,
\end{aligned}
$$

where the $\left|\phi^{\prime}\right\rangle$ and $|\phi\rangle$ states comprise a deuteron formed from nucleons 2, 3 and a free neutron 1 . These states can be projected onto Jacobi momentum eigenstates (with particle 1 being the spectator, $\boldsymbol{p}$ related to the relative motion of particles 2 and 3 , and $\boldsymbol{q}$ related to the relative motion of particle 1 with respect to the c.m. of the $2-3$ subsystem) to give

$$
\langle\boldsymbol{p q} \mid \phi\rangle \propto \delta^{3}\left(\boldsymbol{q}-\boldsymbol{q}_{i}\right)
$$

and

$$
\left\langle\boldsymbol{p} \boldsymbol{q} \mid \phi^{\prime}\right\rangle \propto \delta^{3}\left(\boldsymbol{q}-\boldsymbol{q}_{f}\right) .
$$

Further, $\left|\phi_{0}\right\rangle$ in (6) is the final state of the breakup reaction with three free nucleons - two neutrons and one proton:

$$
\left\langle\boldsymbol{p} \boldsymbol{q} \mid \phi_{0}\right\rangle \propto \delta^{3}\left(\boldsymbol{p}-\boldsymbol{p}^{f}\right) \delta^{3}\left(\boldsymbol{q}-\boldsymbol{q}^{f}\right) .
$$

In the above relations $\boldsymbol{q}_{i}$ and $\boldsymbol{q}_{f}$ are the center-of-mass momenta of the free particle in the initial and final states 
of the elastic scattering process, respectively, and $\boldsymbol{p}^{f}, \boldsymbol{q}^{f}$ are the Jacobi momenta describing the final state in the breakup process with three free particles. The aim of this paper is to provide a numerical realization of the calculations from [19], however, since we now deal with two final channels, we chose a bit different notation for the kinematical variables. Momentum vectors related to the elastic scattering process will be marked using lower indexes " $i$ " and " $f$ " for the initial and final state, respectively. For the breakup process upper indexes " $i$ " and "f " will be used. More details on the kinematics will be given in sect. 3.1 and in fig. 1. The isospin-spin structure of the final and initial states will be described in detail in sect. 3.2.

Equation (5) can be iterated and written in the form of a series:

$$
\check{T}=\check{t} \check{P}+\check{t} \check{G}_{0} \check{P} \check{t} \check{P}+\check{t} \check{G}_{0} \check{P} \check{t} \check{G} \check{G}_{0} \check{P} \check{t} \check{P}+\ldots
$$

In this paper we restrict ourselves only to the first-order term of this expansion, i.e. we approximate $\check{T}$ by the driving term:

$$
\check{T} \approx \check{t} \check{P}
$$

The consequence of using such an approximation is a new, simplified form of the breakup and elastic scattering transition operators from eqs. (6) and (7), $\check{u}_{0}$ and $\check{u}$ :

$$
\left\langle\phi_{0}\left|\check{u}_{0}\right| \phi\right\rangle=\left\langle\phi_{0}|(1+\check{P}) \check{t} \check{P}| \phi\right\rangle
$$

and

$$
\left\langle\phi^{\prime}|\check{u}| \phi\right\rangle=\left\langle\phi^{\prime}\left|\check{P} \check{G}_{0}^{-1}+\check{P} \check{t} \check{P}\right| \phi\right\rangle .
$$

Following [20,21], in a numerical realization of the calculations we will use the "three-dimensional" approach. In this method we work with the three-dimensional degrees of freedom of the nucleons directly and thus avoid the decomposition of operators into partial waves. This makes the calculations flexible and allows an easy incorporation of new models of $2 \mathrm{~N}$ interactions into the calculations. The method outlined in $[20,21]$ and used in this paper to calculate the $2 \mathrm{~N}$ transition operator and deuteron bound state utilizes a general parity, time reversal and rotation symmetric form of the nucleon-nucleon interaction. Using this form of the potential is equivalent to using "all" partial waves, however, the practical realization is limited by the finite computer resources. Nonetheless it is expected that numerical results obtained using the traditional PWD approach will converge to the $3 \mathrm{D}$ results if an increasing number of partial waves is taken into account.

From the practical side, after the momentum space projection, all calculations in the $3 \mathrm{D}$ formalism are reduced to simple matrix equations as described in sect. 3.1. The matrix representation of all operators that are introduced in this section can be easily calculated using our software for the Mathematica ${ }^{\circledR}$ system [23]. In particular, the treatment of particle permutations is greatly simplified in comparison to the PWD approach. Construction of the matrix representation of the permutation operators in the spin and isospin spaces of the three particles is described in sect. 3.4.
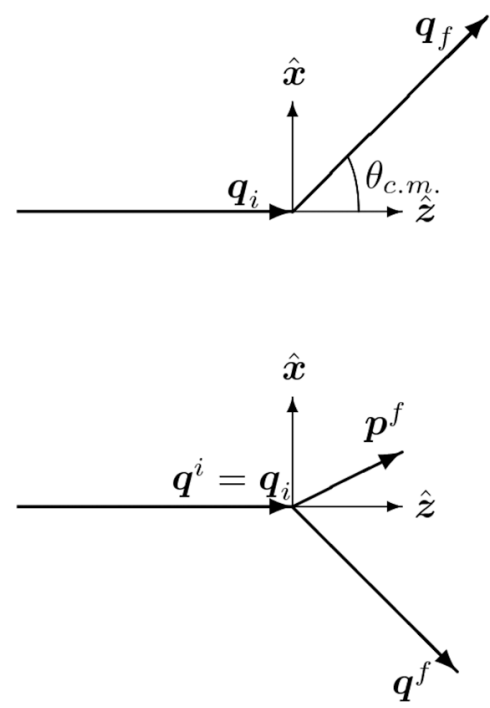

Fig. 1. Top panel: description of kinematic for elastic scattering. Initially the neutron has momentum $\boldsymbol{q}_{i}$ in the $\hat{\boldsymbol{z}}$-direction. In the final state, after interacting with the deuteron, the neutron receives momentum $\boldsymbol{q}_{f}$. Bottom panel: kinematic situation for the breakup process. The momentum of the incident particle is $\boldsymbol{q}^{i} \equiv \boldsymbol{q}_{i}$. In the final state the Jacobi momenta of three free particles are $\boldsymbol{p}^{f}$ and $\boldsymbol{q}^{f}$.

\section{Formal ingredients}

In subsect. 3.1 we give the momentum-space-projected versions of eqs. (13) and (14). Our calculations are performed using the isospin formalism in which the proton and neutron are two charge states of the same particle, the spin $\frac{1}{2}$ and isospin $\frac{1}{2}$ nucleon. Thus, any isospin-spin operator in the $3 \mathrm{~N}$ system can be represented, after the momentum space projection, as a $64 \times 64$ matrix.

The choice to work with $64 \times 64$ matrices directly was made out of convenience. The alternative approach is to separate the spin and isospin cases but this makes the analytical calculations involving multiple permutation operators significantly more complicated.

In the subsections that follow, subsects. 3.2, 3.3 and 3.4, we outline the construction of all relevant matrix elements in terms of $64 \times 64$ matrices and 64 dimensional vectors in the full isospin-spin space of the $3 \mathrm{~N}$ system.

\subsection{Momentum space representation}

The kinematical variables in the center-of-mass frame are depicted in fig. 1. We consider the breakup and elastic channels of the nd scattering process with the initial relative, with respect to the deuteron, momentum of the nucleon $\boldsymbol{q}_{\boldsymbol{i}}$ in the $\hat{\boldsymbol{z}}$-direction. This together with the on-shell condition limits the possible kinematics of the final state for both channels. For the elastic process, the final state is uniquely determined by the center-of-mass scattering angle $\theta_{\text {c.m. }}$. and the magnitudes of the final and initial neutron momenta are equal $\left|\boldsymbol{q}_{\boldsymbol{f}}\right|=\left|\boldsymbol{q}_{\boldsymbol{i}}\right|$. For the breakup 
process we use Jacobi momenta $\boldsymbol{p}^{f}, \boldsymbol{q}^{f}$ (with particle 1 being the spectator, $\boldsymbol{p}^{f}$ related to the relative motion of particles 2 and 3 , and $\boldsymbol{q}^{f}$ related to the relative motion of particle 1 with respect to the c.m. of the $2-3$ subsystem). The final kinematics can, as in the elastic case, be uniquely determined by one real parameter if the directions of momenta of two particles in the final state are fixed. This follows from the on-shell condition:

$$
\frac{\left|\boldsymbol{p}^{f}\right|^{2}}{m}+\frac{3\left|\boldsymbol{q}^{f}\right|^{2}}{4 m}=\frac{3\left|\boldsymbol{q}_{i}\right|^{2}}{4 m}-\left|E_{d}\right|,
$$

where $m$ is the nucleon mass and $E_{d}<0$ is the deuteron binding energy. The parametrization " $S$ " fully determines the kinematics we will use a convention that is described in sect. 2.4 .1 of ref. [1].

The momentum-space-projected versions of eqs. (13) and (14) are worked out by using the completeness relation:

$$
\int \mathrm{d}^{3} \boldsymbol{p} \mathrm{d}^{3} \boldsymbol{q}|\boldsymbol{p} \boldsymbol{q}\rangle\langle\boldsymbol{p} \boldsymbol{q}|=\check{1},
$$

where $|\boldsymbol{p q}\rangle$ is a product state of Jacobi momentum eigenstates normalized as

$$
\left\langle\boldsymbol{p}^{\prime} \boldsymbol{q}^{\prime} \mid \boldsymbol{p} \boldsymbol{q}\right\rangle=\delta^{3}\left(\boldsymbol{p}^{\prime}-\boldsymbol{p}\right) \delta^{3}\left(\boldsymbol{q}^{\prime}-\boldsymbol{q}\right) .
$$

The momenta $\boldsymbol{p}, \boldsymbol{q}$ are chosen so that particle 1 (neutron, projectile in the initial state) is the spectator and $\boldsymbol{p}$ describes the relative momentum of particles 2,3 (deuteron in $\left.\left|\phi^{\prime}\right\rangle,|\phi\rangle\right)$.

We first focus on the matrix elements necessary to calculate the breakup scattering amplitude (13). Inserting the identity operator (16) twice into (13), before the initial and after the final state yields the momentum-spaceprojected expression:

$$
\begin{aligned}
& \left\langle\phi_{0}\left|\check{u}_{0}\right| \phi\right\rangle= \\
& \int \mathrm{d}^{3} \boldsymbol{p}\left(\left\langle\phi_{0} \mid \boldsymbol{p}^{f} \boldsymbol{q}^{f}\right\rangle\left\langle\boldsymbol{p}^{f} \boldsymbol{q}^{f}\left|\check{t}_{12} \check{P}_{23}\right| \boldsymbol{p} \boldsymbol{q}_{i}\right\rangle\left\langle\boldsymbol{p} \boldsymbol{q}_{i} \mid \phi\right\rangle\right. \\
& +\left\langle\phi_{0} \mid \boldsymbol{p}^{f} \boldsymbol{q}^{f}\right\rangle\left\langle\boldsymbol{p}^{f} \boldsymbol{q}^{f}\left|\check{t}_{13} \check{P}_{23}\right| \boldsymbol{p} \boldsymbol{q}_{i}\right\rangle\left\langle\boldsymbol{p} \boldsymbol{q}_{i} \mid \phi\right\rangle \\
& +\left\langle\phi_{0} \mid \boldsymbol{p}^{f} \boldsymbol{q}^{f}\right\rangle\left\langle\boldsymbol{p}^{f} \boldsymbol{q}^{f}\left|\check{P}_{12} \check{P}_{23} \check{\mathrm{P}}_{12} \check{P}_{23}\right| \boldsymbol{p} \boldsymbol{q}_{i}\right\rangle\left\langle\boldsymbol{p} \boldsymbol{q}_{i} \mid \phi\right\rangle \\
& +\left\langle\phi_{0} \mid \boldsymbol{p}^{f} \boldsymbol{q}^{f}\right\rangle\left\langle\boldsymbol{p}^{f} \boldsymbol{q}^{f}\left|\check{P}_{12} \check{P}_{23} \check{t}_{13} \check{P}_{23}\right| \boldsymbol{p} \boldsymbol{q}_{i}\right\rangle\left\langle\boldsymbol{p} \boldsymbol{q}_{i} \mid \phi\right\rangle \\
& +\left\langle\phi_{0} \mid \boldsymbol{p}^{f} \boldsymbol{q}^{f}\right\rangle\left\langle\boldsymbol{p}^{f} \boldsymbol{q}^{f}\left|\check{P}_{13} \check{P}_{23} \check{\mathrm{P}}_{12} \check{P}_{23}\right| \boldsymbol{p} \boldsymbol{q}_{i}\right\rangle\left\langle\boldsymbol{p} \boldsymbol{q}_{i} \mid \phi\right\rangle \\
& \left.+\left\langle\phi_{0} \mid \boldsymbol{p}^{f} \boldsymbol{q}^{f}\right\rangle\left\langle\boldsymbol{p}^{f} \boldsymbol{q}^{f}\left|\check{P}_{13} \check{P}_{23} \check{\mathrm{P}}_{13} \check{P}_{23}\right| \boldsymbol{p} \boldsymbol{q}_{i}\right\rangle\left\langle\boldsymbol{p} \boldsymbol{q}_{i} \mid \phi\right\rangle\right)
\end{aligned}
$$

In the above equation $\left\langle\boldsymbol{p}^{f} \boldsymbol{q}^{f} \mid \phi_{0}\right\rangle$ and $\left\langle\boldsymbol{p} \boldsymbol{q}_{i} \mid \phi\right\rangle$ are the momentum-space-projected final and initial states. They are represented by vectors in the joined isospin-spin space and their structure will be discussed in the next section. Since the projectile momentum $\boldsymbol{q}_{i}$ in the initial state as well as $\boldsymbol{q}^{f}$ and $\boldsymbol{p}^{f}$ in the final state are specified, three integrations from (16) can be eliminated. The only remaining integral in (18) stems from the relative $2 \mathrm{~N}$ momentum in the deuteron (initial state) and can be eliminated using the property of the two particle transition operator in $3 \mathrm{~N}$ momentum space:

$$
\begin{aligned}
& \left\langle\boldsymbol{p}^{\prime} \boldsymbol{q}^{\prime}|\check{t}(E)| \boldsymbol{p} \boldsymbol{q}\right\rangle=\delta^{3}\left(\boldsymbol{q}^{\prime}-\boldsymbol{q}\right) \\
& \left\langle\boldsymbol{p}^{\prime}\left|\check{t}^{2 N}\left(E^{2 N}=E-\frac{3}{4 m} \boldsymbol{q}^{2}\right)\right| \boldsymbol{p}\right\rangle,
\end{aligned}
$$

where $\left\langle\boldsymbol{p}^{\prime}\left|\check{t}^{2 N}\right| \boldsymbol{p}\right\rangle$ is the matrix element of the transition operator between the $2 \mathrm{~N}$ relative momentum eigenstates of particles 2,3 and $E-\frac{3}{4 m} \boldsymbol{q}^{2}$ is the $2 \mathrm{~N}$ subsystem energy.

The momentum space permutations in (18) can be carried out using (see, e.g., $[19,22])$ :

$$
\begin{aligned}
\check{P}_{12}|\boldsymbol{p} \boldsymbol{q}\rangle & =\left|\frac{1}{4}(2 \boldsymbol{p}+3 \boldsymbol{q}) \boldsymbol{p}-\frac{\boldsymbol{q}}{2}\right\rangle, \\
\check{P}_{23}|\boldsymbol{p} \boldsymbol{q}\rangle & =|-\boldsymbol{p} \boldsymbol{q}\rangle, \\
\check{P}_{13}|\boldsymbol{p} \boldsymbol{q}\rangle & =\left|\frac{1}{4}(2 \boldsymbol{p}-3 \boldsymbol{q})-\boldsymbol{p}-\frac{\boldsymbol{q}}{2}\right\rangle .
\end{aligned}
$$

Considering all the permutation cases from (18) or similar equations is a meticulous task. We gather all the relevant resulting expressions in appendix A for the readers' convenience. This way, below, we can focus on the isospin-spin structure of the final expressions for the scattering amplitudes.

After carrying out all the momentum space permutations using (20)-(22) and the application of properties (17), (19) the final expression for the breakup scattering amplitude can be written as

$$
\begin{aligned}
\left\langle\phi_{0}\left|\check{u}_{0}\right| \phi\right\rangle= & \sum_{c=1}^{6}\left[\left\langle\boldsymbol{p}^{f} \boldsymbol{q}^{f} \mid \phi_{0}\right\rangle\right]^{\dagger}\left[\check{P}_{c}^{\mathrm{BL}}\right] \\
& {\left[\left\langle\boldsymbol{p}_{c}^{\mathrm{BL}}\left|t^{2 N}\left(E-\frac{3}{4 m}\left|\boldsymbol{q}_{c}^{\mathrm{BQ}}\right|^{2}\right)\right| \boldsymbol{p}_{c}^{\mathrm{BR}}\right\rangle\right] } \\
& {\left[\check{P}_{c}^{\mathrm{BR}}\right]\left[\left\langle\boldsymbol{p}_{c}^{\mathrm{BD}} \boldsymbol{q}_{i} \mid \phi\right\rangle\right], }
\end{aligned}
$$

where index "c" enumerates the six permutation cases from eq. (18):

$$
\begin{aligned}
& (\check{1}+\check{P}) \check{t} \check{P}=\check{t} \check{P}_{12} \check{P}_{23}+\check{t} \check{P}_{13} \check{P}_{23}+\check{P}_{12} \check{P}_{23} \check{t} \check{P}_{12} \check{P}_{23} \\
& +\check{P}_{12} \check{P}_{23} \check{t}_{13} \check{P}_{23}+\check{P}_{13} \check{P}_{23} \check{t} \check{P}_{12} \check{P}_{23}+\check{P}_{13} \check{P}_{23} \check{t} \check{P}_{13} \check{P}_{23}
\end{aligned}
$$

and all objects inside square brackets [...] are isospin-spin operators or states, represented by $64 \times 64$ matrices or 64 dimensional vectors, respectively. The vectors $\left[\left\langle\boldsymbol{p}^{f} \boldsymbol{q}^{f}\right|\right.$ $\left.\left.\phi_{0}\right\rangle\right]$ and $\left[\left\langle\boldsymbol{p}_{c}^{\mathrm{BD}} \boldsymbol{q}_{i} \mid \phi\right\rangle\right]$ contain the complete information about the spin and isospin of the final and initial state. $\left[\check{P}_{c}^{\mathrm{BL}}\right]$ and $\left[\check{P}_{c}^{\mathrm{BR}}\right]$ are permutation operators, in particular $\left[\check{P}_{1}^{\mathrm{BL}}\right]=\left[\check{P}_{2}^{\mathrm{BL}}\right]=[\check{1}]$. Further,

$$
\left[\left\langle\boldsymbol{p}_{c}^{\mathrm{BL}}\left|t^{2 N}\left(E-\frac{3}{4 m}\left|\boldsymbol{q}_{c}^{\mathrm{BQ}}\right|^{2}\right)\right| \boldsymbol{p}_{c}^{\mathrm{BR}}\right\rangle\right]
$$

is the transition operator matrix and finally $\boldsymbol{p}_{c}^{\mathrm{BL}}, \boldsymbol{p}_{c}^{\mathrm{BR}}$, $\boldsymbol{q}_{c}^{\mathrm{BQ}}$ and $\boldsymbol{p}_{c}^{\mathrm{BD}}$ are momenta that result from the application of the permutations. A complete list of permutation operators and momenta necessary to construct (23) for every case " $c$ " is given in tables 1 and 2 in appendix A. The transition operator and the deuteron bound state require special attention and will be discussed separately in subsects. 3.2 and 3.3. The explicit form of the particle exchange operators will be discussed in 3.4.

The expression for the elastic transition amplitude (14) is more complex. Let us consider two parts of this expression separately,

$$
a_{1}^{\mathrm{EL}}=\left\langle\phi^{\prime}\left|\check{P} \check{G}_{0}^{-1}\right| \phi\right\rangle
$$


and

$$
a_{2}^{\mathrm{EL}}=\left\langle\phi^{\prime}|\check{P} \check{t} \check{P}| \phi\right\rangle,
$$

so $\left\langle\phi^{\prime}|\check{u}| \phi\right\rangle=a_{1}^{\mathrm{EL}}+a_{2}^{\mathrm{EL}}$. As in the case of the breakup scattering amplitude, we will construct the amplitude in terms of $64 \times 64$ matrices and 64 dimensional vectors.

The amplitude $a_{1}^{\mathrm{EL}}$ takes a simple form. The momentum space projected expression originates from inserting the completeness relation (16) after the permutation operator. More details on the final $\left|\phi^{\prime}\right\rangle$ and initial $|\phi\rangle$ states will be presented in subsect. 3.2 but it is not surprising that they are constructed from a linear combination of states with different relative momenta of particles 2 and 3 that form a bound state. This can potentially introduce two three-fold integrations, however since

$$
\check{G}_{0}^{-1}|\boldsymbol{p} \boldsymbol{q}\rangle=\left(E-\frac{\boldsymbol{p}^{2}}{m}+\frac{3 \boldsymbol{q}^{2}}{4 m}\right)|\boldsymbol{p q}\rangle,
$$

the $2 \mathrm{~N}$ relative momentum in the deuteron is fully determined by $\boldsymbol{q}_{i}$ and $\boldsymbol{q}_{f}$. Thus the amplitude $a_{1}^{\text {EL }}$ has the form

$$
\begin{aligned}
a_{1}^{\mathrm{EL}}= & \sum_{c=1}^{2}\left(E-\frac{\left|\boldsymbol{p}_{c}^{\mathrm{ER}}\right|^{2}}{m}+\frac{3\left|\boldsymbol{q}_{i}\right|^{2}}{4 m}\right) \\
& {\left[\left\langle\boldsymbol{p}_{c}^{\mathrm{EL}} \boldsymbol{q}_{f} \mid \phi^{\prime}\right\rangle\right]^{\dagger}\left[\check{P}_{c}^{\mathrm{E}}\right]\left[\left\langle\boldsymbol{p}_{c}^{\mathrm{ER}} \boldsymbol{q}_{i} \mid \phi\right\rangle\right] }
\end{aligned}
$$

and the index " $c$ " takes on only two values since there are only two possible permutation in eq. (24) related to the two terms in

$$
\check{P} \check{G}_{0}^{-1}=\check{P}_{12} \check{P}_{23} \check{G}_{0}^{-1}+\check{P}_{13} \check{P}_{23} \check{G}_{0}^{-1} .
$$

The complete information on the permutation operators and momenta for above cases is given in tables 3 and 4 from appendix A.

The amplitude $a_{2}^{\mathrm{EL}}$ is more demanding but the method of calculation is very similar to (23). In this case, since there is a deuteron bound state both in the initial and in the final state, only one three-fold integral remains:

$$
\begin{aligned}
a_{2}^{\mathrm{EL}}= & \sum_{c=1}^{4} \int \mathrm{d}^{3} \boldsymbol{q} \\
& {\left[\left\langle\boldsymbol{p}_{c}^{\mathrm{EDL}} \boldsymbol{q}_{f} \mid \phi^{\prime}\right\rangle\right]^{\dagger}\left[\check{P}_{c}^{\mathrm{EL}}\right] } \\
& {\left[\left\langle\boldsymbol{p}_{c}^{\mathrm{ETL}}\left|t^{2 N}\left(E-\frac{3}{4 m} \boldsymbol{q}^{2}\right)\right| \boldsymbol{p}_{c}^{\mathrm{ETR}}\right\rangle\right] } \\
& {\left[\check{P}_{c}^{\mathrm{ER}}\right]\left[\left\langle\boldsymbol{p}_{c}^{\mathrm{EDR}} \boldsymbol{q}_{i} \mid \phi\right\rangle\right] . }
\end{aligned}
$$

This time the index " $c$ " takes on four values that are related to the four terms in (25):

$$
\begin{aligned}
& \check{P} \check{t} \check{P}=\check{P}_{12} \check{P}_{23} \check{t} \check{P}_{12} \check{P}_{23}+\check{P}_{12} \check{P}_{23} \check{t}_{13} \check{P}_{23} \\
& +\check{P}_{13} \check{P}_{23} \check{t}_{12} \check{P}_{23}+\check{P}_{13} \check{P}_{23} \check{t}_{13} \check{P}_{23} \text {. }
\end{aligned}
$$

These terms correspond directly to four of the six terms from eq. (18). All momenta and permutation operators, for each case " $c$ ", are gathered in tables 5 and 6 in appendix A. It should be stressed that for the amplitude (27) there are different possible choices for the remaining integral. The choice made in (27) allows us to treat the singularity of the $2 \mathrm{~N}$ transition operator at the deuteron bound state energy using the standard subtraction method (see sect. 3.3).

Looking at the forms of the final expressions (23), (26) and (27) it becomes apparent why our choice to work in the full 64 dimensional isospin-spin space is advantageous. Not separating the permutation operators into their spin and isospin components, we can use very straightforward equations that require only matrix manipulations. That being said one should also note the disadvantage of this approach which is an increase in computing time required for the calculations.

\subsection{The deuteron and $3 \mathrm{~N}$ states}

Following $[20,24]$ the deuteron bound state projected on a state with the relative momentum of the two nucleons $\boldsymbol{p}$ can be written in an operator form,

$$
\left\langle\boldsymbol{p} \mid \phi_{d}\left(m_{d}\right)\right\rangle=\sum_{l=1}^{2} \phi_{l}(|\boldsymbol{p}|) \check{b}_{l}(\boldsymbol{p})\left|1 m_{d}\right\rangle,
$$

where $\left|1 m_{d}\right\rangle$ is a $2 \mathrm{~N}$ state with the total spin 1 and its projection $m_{d}, \check{b}_{l}(\boldsymbol{p})$ are $2 \mathrm{~N}$ spin operators listed for example in [20]. The isospin state of the system is $|00\rangle$. Using the $3 \mathrm{D}$ approach, the task of finding the deuteron bound state, for a given $2 \mathrm{~N}$ interaction, is transformed into a system of linear equations, in which energy-dependent operators act on the scalar functions $\phi_{1}(|\boldsymbol{p}|), \phi_{2}(|\boldsymbol{p}|)$ from (28). This is achieved by eliminating the spin degrees of freedom in the integral, time-independent Schrödinger equation and introducing a suitable grid of $|\boldsymbol{p}|$ points. The scalar functions $\phi_{1}(|\boldsymbol{p}|), \phi_{2}(|\boldsymbol{p}|)$ are directly related to the $s$ and $d$ wave components of the deuteron wave function (as given explicitly in $[24,20]$ ) and are a necessary ingredient in the construction of the initial and final states for the nd scattering amplitudes.

The operator form of the deuteron bound state can be used to work out the form of the final $\left|\phi^{\prime}\right\rangle$ and initial $|\phi\rangle$ state. In both cases only an additional free neutron with isospin $\left|\frac{1}{2}-\frac{1}{2}\right\rangle$ and spin $\left|\frac{1}{2} m_{n}\right\rangle$ has to be added. The 64 dimensional vector, containing the complete information on the initial and final spin and isospin of the system, can be constructed in a straightforward way. The initial and final states, projected onto a Jacobi momentum eigenstate $|\boldsymbol{p q}\rangle$ can be written as

$$
\begin{aligned}
& {[\langle\boldsymbol{p} \boldsymbol{q} \mid \phi\rangle]=} \\
& \delta^{3}\left(\boldsymbol{q}-\boldsymbol{q}_{i}\right) \sum_{l=1}^{2} \phi_{l}(|\boldsymbol{p}|)\left[B_{l}(\boldsymbol{p})\right]\left[\left|m_{n} m_{d}\right\rangle\right] \\
& {\left[\left\langle\boldsymbol{p} \boldsymbol{q} \mid \phi^{\prime}\right\rangle\right]=} \\
& \delta^{3}\left(\boldsymbol{q}-\boldsymbol{q}_{f}\right) \sum_{l=1}^{2} \phi_{l}(|\boldsymbol{p}|)\left[B_{l}(\boldsymbol{p})\right]\left[\left|m_{n}^{\prime} m_{d}^{\prime}\right\rangle\right],
\end{aligned}
$$


where $m_{n}$ is the neutron spin projection and $m_{d}$ is the deuteron spin projection. The matrices and vectors in (29), (30) can be constructed by using the Kronecker product $\otimes$ to implement the tensor product of operators and states. Using this method, $\left[\left|m_{n} m_{d}\right\rangle\right]$ and $\left[B_{l}(\boldsymbol{p})\right]$ have the following forms:

$$
\begin{aligned}
& {\left[\left|m_{n} m_{d}\right\rangle\right]^{64}=} \\
& \left(\left[\left|\frac{1}{2}-\frac{1}{2}\right\rangle\right]^{2} \otimes[|00\rangle]^{4}\right)^{3 N \text { isospin }} \\
& \otimes\left(\left[\left|\frac{1}{2} m_{n}\right\rangle\right]^{2} \otimes\left[\left|1 m_{d}\right\rangle\right]^{4}\right)^{3 N \text { spin }}
\end{aligned}
$$

and

$$
\begin{aligned}
& {\left[B_{l}(\boldsymbol{p})\right]^{64 \times 64}=} \\
& \left([\check{1}]^{2 \times 2} \otimes[\check{1}]^{4 \times 4}\right)^{3 N \text { isospin }} \\
& \otimes\left([\check{1}]^{2 \times 2} \otimes\left[\check{b}_{l}(|\boldsymbol{p}|)\right]^{4 \times 4}\right)^{3 N \text { spin }} .
\end{aligned}
$$

In (31) and (32) single nucleon states and operators are represented by 2 dimensional vectors $[\ldots]^{2}$ and $2 \times 2$ matrices $[\ldots]^{2 \times 2}$, respectively. $2 \mathrm{~N}$ states and operators are represented by 4 dimensional vectors [... $]^{4}$ and $4 \times 4$ matrices $[\ldots]^{4 \times 4}$. In particular $[1]^{2 \times 2}$ is an identity operator in the $2 \mathrm{~N}$ spin (isospin) space. Additionally, the expressions have the $3 \mathrm{~N}$ isospin and spin spaces marked separately.

All calculations that will be presented here are done with the Bonn B [5] potential and the deuteron bound state calculations are performed according to [20]. This approach results in the deuteron binding energy $E_{b}=$ $-2.2242 \mathrm{MeV}$. The calculation scheme that we developed is, however, flexible and can be applied also to other models of nuclear forces.

\subsection{The two-nucleon transition operator}

The $2 \mathrm{~N}$ transition operator $\check{t}_{i}(E)$ is the most crucial element of our calculations. It was introduced in eq. (2) where it was written in the full $3 \mathrm{~N}$ space. The presence of only one $2 \mathrm{~N}$ interaction operator $\check{V}:=\check{V}_{1}$ in (2) makes it possible to limit the calculations to the $2 \mathrm{~N}$ space. The relation between the two particle transition operator calculated in $3 \mathrm{~N}$ space and the two particle transition operator calculated in $2 \mathrm{~N}$ space was given in (19) and in this subsection we will focus on the latter.

Using the methods from [20-22] allows for an easy incorporation of new models of $2 \mathrm{~N}$ interactions. This useful feature is also an important motivation behind the application of the 3D approach to nd scattering. A detailed description of the transition operator calculations can be found in one of our earlier papers [20-22] and below we give only short description of these calculations.

The starting point is the general, parity and time reversal symmetric form of the $2 \mathrm{~N}$ potential [25]. In this form, the $2 \mathrm{~N}$ interaction is defined by the scalar functions $v_{i}^{\gamma}$ via

$$
\begin{aligned}
& \left\langle\boldsymbol{p}^{\prime}|\check{V}| \boldsymbol{p}\right\rangle= \\
& \sum_{i=1}^{6} \sum_{\gamma} v_{i}^{\gamma}\left(\left|\boldsymbol{p}^{\prime}\right|,|\boldsymbol{p}|, \hat{\boldsymbol{p}^{\prime}} \cdot \hat{\boldsymbol{p}}\right)|\gamma\rangle\langle\gamma| \otimes \check{w}_{i}\left(\boldsymbol{p}^{\prime}, \boldsymbol{p}\right),
\end{aligned}
$$

where $|\gamma\rangle$ is one of the 4 possible isospin states of the $2 \mathrm{~N}$ system, $\boldsymbol{p}^{\prime}, \boldsymbol{p}$ are the relative $2 \mathrm{~N}$ momenta and $\check{w}_{i}\left(\boldsymbol{p}^{\prime}, \boldsymbol{p}\right)$ are known $2 \mathrm{~N}$ spin operators (they are listed, for example, in [20]). The same symmetry considerations that lead to (33) can also be applied to the $2 \mathrm{~N}$ transition operator. It can be defined by the energy-dependent scalar functions $t_{i}^{\gamma}(E)$ via

$$
\begin{aligned}
& \left\langle\boldsymbol{p}^{\prime}|\check{t}(E)| \boldsymbol{p}\right\rangle= \\
& \sum_{i=1}^{6} \sum_{\gamma} t_{i}^{\gamma}\left(E ;\left|\boldsymbol{p}^{\prime}\right|,|\boldsymbol{p}|, \hat{\boldsymbol{p}^{\prime}} \cdot \hat{\boldsymbol{p}}\right)|\gamma\rangle\langle\gamma| \otimes \check{w}_{i}\left(\boldsymbol{p}^{\prime}, \boldsymbol{p}\right) .
\end{aligned}
$$

In the next step decompositions (33) and (34) are inserted into the Lippmann-Schwinger equation (2) and the spin dependencies are removed. The final result is a set of linear equations, in which energy-dependent operators act on the scalar functions $t_{i}^{\gamma}(E)$. In order to find the solution, the functions $t_{i}^{\gamma}\left(E ;\left|\boldsymbol{p}^{\prime}\right|,|\boldsymbol{p}|, \hat{\boldsymbol{p}^{\prime}} \cdot \hat{\boldsymbol{p}}\right)$ first need to be discretized over a given mesh of $\left|\boldsymbol{p}^{\prime}\right|,|\boldsymbol{p}|, \hat{\boldsymbol{p}}^{\prime} \cdot \hat{\boldsymbol{p}}$ points, effectively turning them into vectors. Next it is possible to use various approaches. One approach requires the construction of a matrix representation of the energy-dependent operators and the direct solution of the resulting matrix equation. Another, more simple, approach utilizes the Arnoldi algorithm [26] and requires only the numerical implementation of the action of the energy-dependent operators on the scalar functions. Both approaches were explored in [20-22] but the present results, given here in sect. 4, are obtained using the second method. It should be stressed out that the final operator equations take on a different form for positive and negative $2 \mathrm{~N}$ energy cases.

In order to construct the amplitudes (23) and (27), the transition operator must be calculated for a wide range of $2 \mathrm{~N}$ energies, both positive and negative. The task of computing the appropriate scalar functions is numerically very demanding. We use the computing resources of the Jülich Supercomputing Center to calculate these functions for the wide spectrum of energies required by nd scattering.

A further complication comes from the fact that the transition operator has singular behavior at the deuteron binding energy $E_{d}$ for the isospin $|00\rangle$ case. The form of this pole is well known:

$$
\check{t}_{i}^{|00\rangle}\left(E^{2 N} \rightarrow E_{d}\right) \propto \frac{1}{E^{2 N}-E_{d}+i \epsilon},
$$

and this knowledge can be used to carry out the $\boldsymbol{q}$ integration in (27). A cut-off value $|\boldsymbol{q}|<\bar{q}$ is introduced to this integral and the standard formula using the principal 

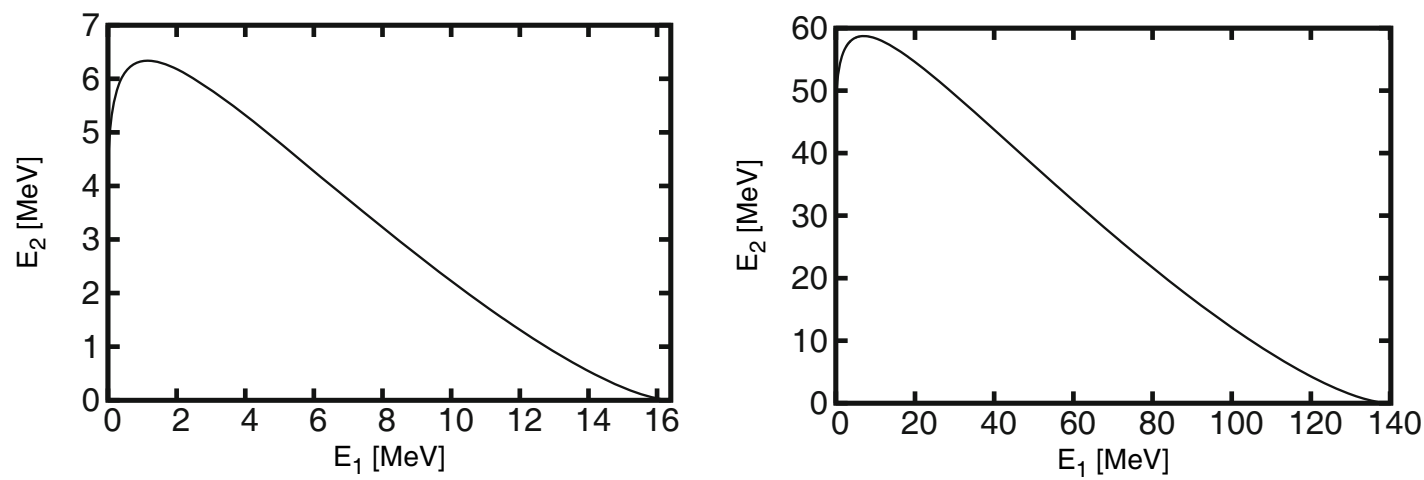

Fig. 2. The energy of the first neutron (exiting the reaction at $\theta_{1}=30^{\circ}$ with respect to the $\hat{\boldsymbol{z}}$-axes) $E_{1}$ and the second neutron (exiting the reaction at $\theta_{2}=60^{\circ}$ with respect to the $\hat{z}$-axes and with the relative azimuthal angle $\phi_{12}=180^{\circ}$ ) $E_{2}$ in the breakup process as a function of the " $S$ " parameter of the kinematics curve for $25 \mathrm{MeV}$ (left panel) and $190 \mathrm{MeV}$ (right panel). For the laboratory energy of $25 \mathrm{MeV}$ the curve starts at $E_{1}=16.4 \mathrm{MeV}$ and $E_{2}=0.0 \mathrm{MeV}$. For the laboratory energy of $190 \mathrm{MeV}$ the curve starts at $E_{1}=140.2 \mathrm{MeV}$ and $E_{2}=0.0 \mathrm{MeV}$.

value can be applied:

$$
\begin{aligned}
& \int_{0}^{\bar{y}} \mathrm{~d} y \frac{y^{2}}{y_{0}^{2}-y^{2}+i \epsilon} f(y)= \\
& \int_{0}^{\bar{y}} \mathrm{~d} y \frac{y^{2} f(y)-y_{0}^{2} f\left(y_{0}\right)}{y_{0}^{2}-y^{2}} \\
& +\frac{1}{2} y_{0} f\left(y_{0}\right)\left(\ln \left(\frac{\bar{y}+y_{0}}{\bar{y}-y_{0}}\right)-i \pi\right) .
\end{aligned}
$$

The choice of the final integration variable used in (27) was made in order to make use of (36). It should be noted that in the above formula, $f(y)$ does not have to return a number. In the practical realization of our calculations it returned a $64 \times 64$ dimensional representation of the operators form (27).

Finally, the $2 \mathrm{~N}$ relative momentum space projected matrix element of the transition operator,

$$
\left\langle\boldsymbol{p}^{\prime}|\check{t}(E)| \boldsymbol{p}\right\rangle,
$$

is an operator in the isospin and spin spaces of the $2 \mathrm{~N}$ system. In order to construct the $64 \times 64$ dimensional matrix representation of this operator in the $3 \mathrm{~N}$ isospin-spin space required by (23) and (27) we simply insert the identity operator $\check{1}$ in both the spin and isospin spaces of particle 1 of (34) in a similar fashion as in eq. (32):

$$
\begin{aligned}
& \left\langle\boldsymbol{p}^{\prime}|\check{t}(E)| \boldsymbol{p}\right\rangle= \\
& \sum_{i=1}^{6} \sum_{\gamma} t_{i}^{\gamma}\left(E ;\left|\boldsymbol{p}^{\prime}\right|,|\boldsymbol{p}|, \hat{\boldsymbol{p}^{\prime}} \cdot \hat{\boldsymbol{p}}\right) \\
& \left([\check{1}]^{2 \times 2} \otimes[|\gamma\rangle\langle\gamma|]^{4 \times 4}\right)^{3 N \text { isospin }} \\
& \otimes\left([\check{1}]^{2 \times 2} \otimes\left[\check{w}_{i}\left(\boldsymbol{p}^{\prime}, \boldsymbol{p}\right)\right]^{4 \times 4}\right)^{3 N \text { spin }} .
\end{aligned}
$$

\subsection{The permutation operators}

The final missing ingredients necessary to obtain the scattering amplitudes are the permutation operators $\left[\check{P}_{i j}\right]$. In order to work out the matrix representation of the particle exchange operators in the spin (isospin) space it is necessary to consider the action of the permutation on individual particle spin (isospin) states. With our choice for the basis from table $7(|\uparrow \uparrow \uparrow\rangle,|\uparrow \uparrow \downarrow\rangle,|\uparrow \downarrow \uparrow\rangle, \ldots,|\downarrow \downarrow \downarrow\rangle)$ the matrix representations are given in eqs. (B.1), (B.2), (B.3) from appendix B for the isospin and spin spaces separately. The $64 \times 64$ matrix representation of the permutation operator in the joined isospin-spin space of the $3 \mathrm{~N}$ system can be easily constructed using the Kronecker product:

$$
\left[P_{i j}\right]^{3 \mathrm{~N}}=\left[P_{i j}\right]^{3 \mathrm{~N} \text { isospin }} \otimes\left[P_{i j}\right]^{3 \mathrm{~N} \text { spin }} .
$$

\section{Numerical results}

As mentioned earlier all 3D results were obtained using the Bonn B potential [5] and are compared with results derived using partial wave decomposition. Using partial waves, convergence is observed as more partial waves are taken into account. However, in some cases a very large number of partial waves is necessary to observe full agreement. Achieving this was, in some cases, difficult due to the large memory requirements of the PWD code. This demonstrates a nice feature of the $3 \mathrm{D}$ approach which is equivalent to taking into account all partial waves. In addition, all 3D results obtained in first order only are compared to values produced with the full equation for the $\check{T}$ operator (5).

Our calculations were performed for two different laboratory energies $25 \mathrm{MeV}$ and $190 \mathrm{MeV}$. For the breakup case we chose a kinematical configuration in which two neutrons in the final state emerge at angles $30^{\circ}$ and $60^{\circ}$ relative to the $\hat{\boldsymbol{z}}$-axes and with the relative azimuthal angle $\phi_{12}=180^{\circ}$. The location of the kinematics in the plane of the kinetic energy of the first $E_{1}$ and second $E_{2}$ particle, for the $25 \mathrm{MeV}$ and $190 \mathrm{MeV}$ cases, is related to the kinematics curve and is plotted in fig. 2. The differential cross sections with respect to the kinematics parameter " $S$ " are presented in figs. 3 and 4 . In fig. 3 we demonstrate 


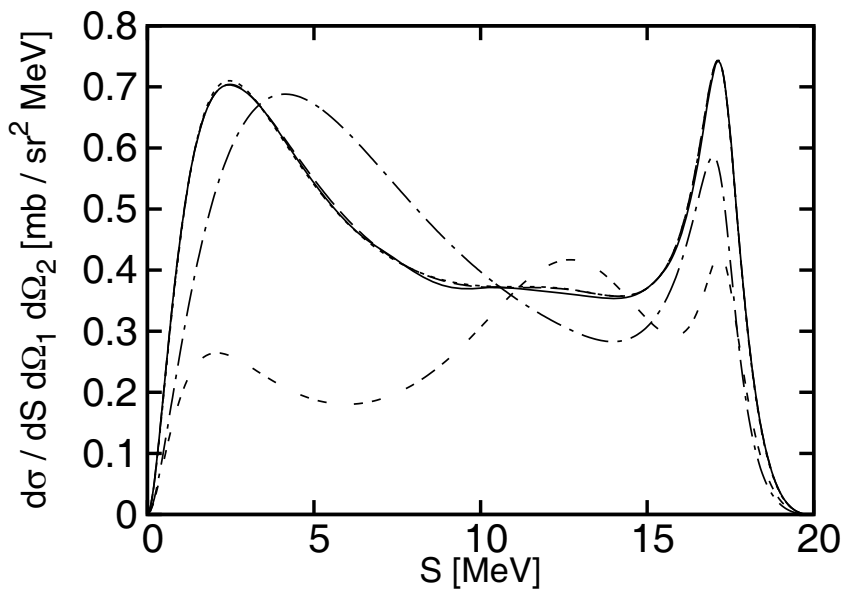

Fig. 3. Differential cross section for the nd breakup process with respect to the kinematics curve parameter " $S$ " (see fig. 2). The results were obtained for laboratory kinetic energy $25 \mathrm{MeV}$. The two neutrons in the final state exit the reaction at $\theta_{1}=30^{\circ}$ and $\theta_{2}=60^{\circ}$ (with the relative azimuthal angle $\phi_{12}=180^{\circ}$ ), respectively. $3 \mathrm{D}$ results are represented by a solid line. The partial wave results, converging to the full $3 \mathrm{D}$ results, obtained with the maximum total angular momentum $J_{\max }=\frac{5}{2}, \frac{15}{2}, \frac{25}{2}$ and the maximum angular momentum of the $2-3$ particle subsystem $j_{\max }=3$, are given by using dashdotted, dotted and dashed lines, respectively. The lines above $J_{\max }=\frac{15}{2}$ merge. PWD results that take into account the full equation for $\breve{T}$ as given in eq. (5) are plotted using the doubledashed line for comparison, they are obtained using $J_{\max }=\frac{25}{2}$ and $j_{\max }=7$.

convergence of the PWD calculations to the $3 \mathrm{D}$ result with respect to increasing total angular momenta. In fig. 4 we demonstrate convergence with respect to increasing angular momentum of the 2-3 subsystem.

We plot selected spin observables for the breakup process, for the same configurations, in figs. 5 and 6 . The plots contain the deuteron and nucleon vector analyzing powers $\left(A_{y}^{d}, A_{y}^{N}\right)$ and the deuteron tensor analyzing powers $\left(A_{x x}\right.$, $\left.A_{y y}, A_{z z}\right)$ [1]. For $25 \mathrm{MeV}$ we observe nice agreement of the PWD calculations with the $3 \mathrm{D}$ results even if only a small number of partial waves is taken into account. For $190 \mathrm{MeV}$ we also observe convergence of the PWD results to the $3 \mathrm{D}$ results, however, in order to get full agreement a larger number of partial waves should be taken into account.

For the elastic nd scattering process excellent agreement and convergence of the PWD results and the 3D results is observed for all investigated observables and energies. Figure 7 contains the differential cross sections for $25 \mathrm{MeV}$ and $190 \mathrm{MeV}$. Figures 8 and 9 contain selected spin observables. In fig. 10 we demonstrate the convergence of the PWD results to the 3D results for $190 \mathrm{MeV}$ not only with the increasing $2-3$ particle subsystem angular momentum but also with respect to the total angular momentum.

All new results presented in this paper use only the driving term of the $3 \mathrm{~N}$ Faddeev equations. Nevertheless,

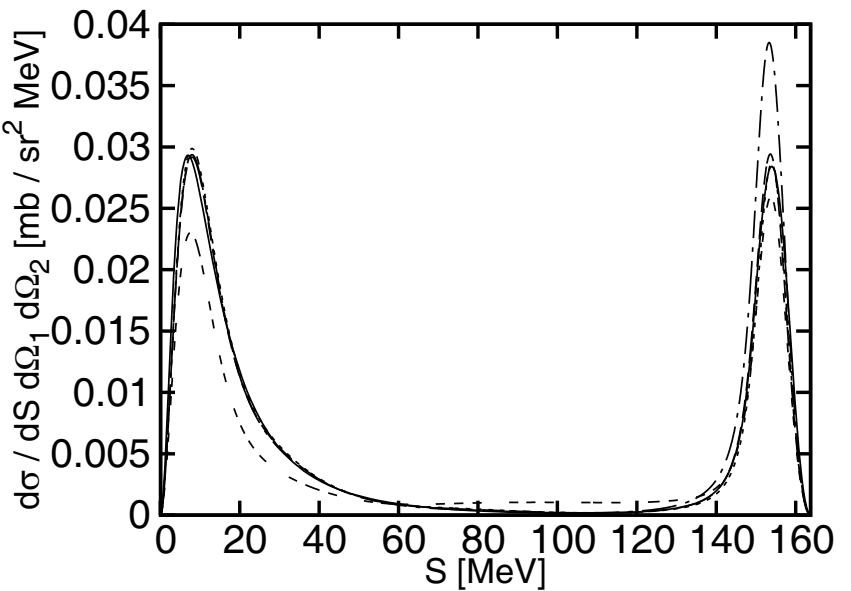

Fig. 4. The same as in fig. 3 but for $190 \mathrm{MeV}$ and $J_{\max }=\frac{25}{2}$. Here the PWD results calculated with $j_{\max }=3,5,8$ are shown using dash-dotted, dotted and dashed lines, respectively. The solid obtained using the 3D approach is practically identical as the dashed, $j_{\max }=8$, result. PWD results that take into account the full equation for $\check{T}$ (5) are plotted using the double dashed line for comparison, they are obtained using $J_{\max }=\frac{25}{2}$ and $j_{\max }=7$.

they are an important step in the development of the full $3 \mathrm{D}$ treatment of $3 \mathrm{~N}$ scattering. We showed that the previously developed 3D techniques for calculating the transition operator and the deuteron bound state $[20-22]$ can be successfully applied to the new problem. These techniques can be applied to any $2 \mathrm{~N}$ potential (however, in this paper we restricted ourselves to the Bonn B force [5]) and are equivalent to using all partial waves. The high precision and flexibility of these calculations could make the new $3 \mathrm{D}$ treatment of $3 \mathrm{~N}$ scattering a valuable tool to verify modern nuclear forces.

The formalism employed in the calculations presented in this paper is closely related to that of [19]. In order to simplify the calculations we decided not to separate the isospin and spin spaces and instead to work directly with the $64 \times 64$ dimensional representation of operators in the joined $3 \mathrm{~N}$ isospin-spin space. This allowed us to consider both the elastic and breakup channels of the nd scattering reaction in a consistent way that requires only simple matrix manipulations. Furthermore, we believe that a similar approach could also be applicable to calculations that involve $3 \mathrm{NFs}$.

\section{Summary}

In this paper we demonstrated that, up to the driving term the "three-dimensional" calculations, of the neutrondeuteron breakup and the elastic scattering processes, are in good agreement with the classical calculations that use partial waves. Convergence of the PWD results to the $3 \mathrm{D}$ results, both in the relative and total angular momentum, is observed. However, for higher energies around $190 \mathrm{MeV}$ a very large number of partial waves is required. Implementing this requirement was difficult due to the high 

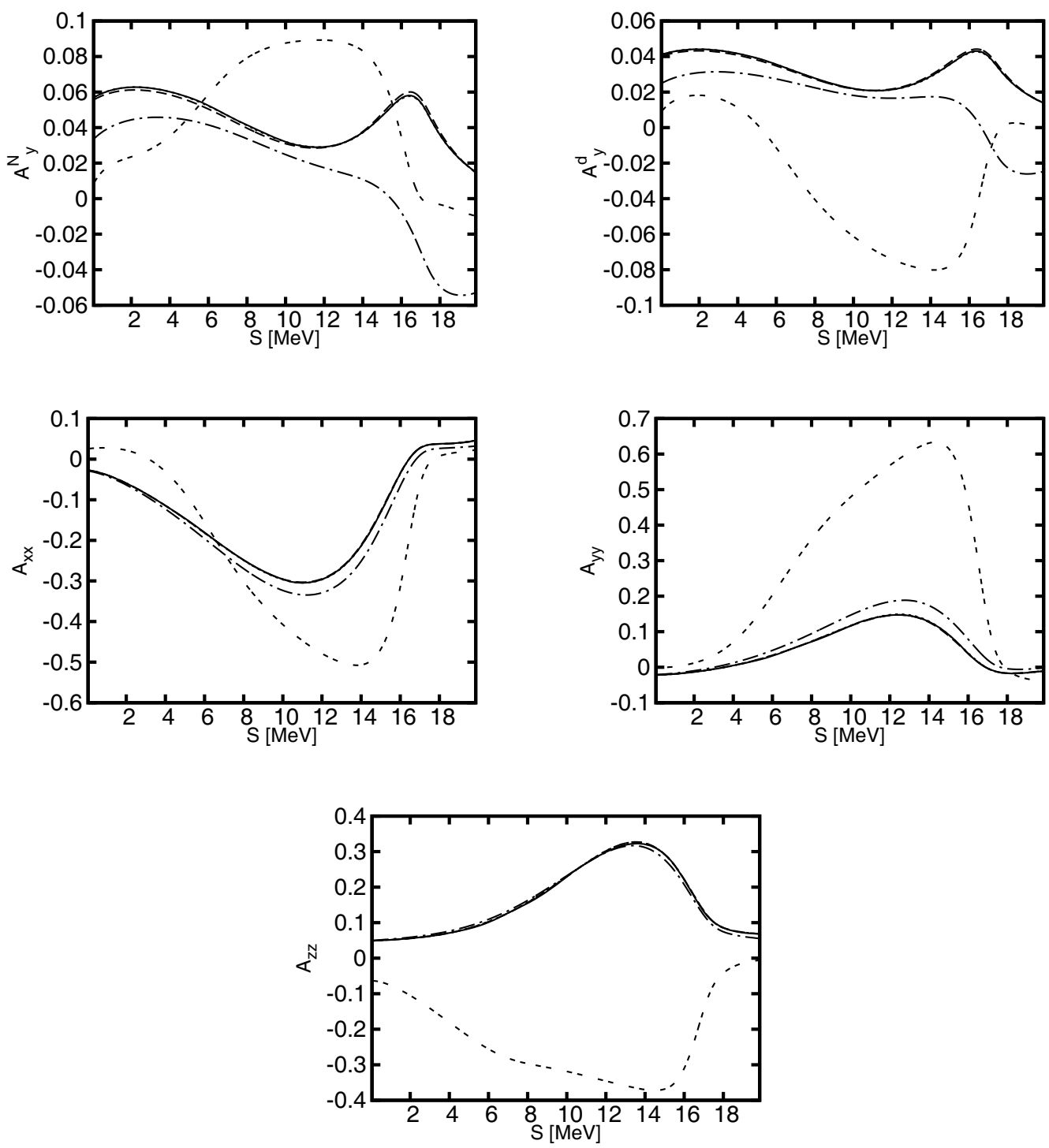

Fig. 5. Similar to fig. 4 but for selected spin observables (for more details see text) for the breakup process at $25 \mathrm{MeV}$ in LAB (two neutrons in the final state exit the reaction at angles $\theta_{1}=30^{\circ}$ and $\theta_{2}=60^{\circ}$ relative to the $\hat{\boldsymbol{z}}$-axes and with the relative azimuthal angle $\phi_{12}=180^{\circ}$ ). The solid lines are calculated for using the 3D formalism. The dash-dotted, dotted and dashed lines are calculated using PWD with $J_{\max }=\frac{25}{2}$ and $j_{\max }=1,3,5$, respectively. The 3D and PWD results merge above $j_{\text {max }}=3$. PWD results that take into account the full equation for $\check{T}(5)$ are plotted using the double-dashed line for comparison, they are obtained using $J_{\max }=\frac{25}{2}$ and $j_{\max }=7$.

memory requirements of the PWD code and demonstrates a nice feature of the $3 \mathrm{D}$ calculations - they take into account "all" partial waves up to infinity. This makes the new approach a very good tool for calculations at higher energies. The drawback of the new approach is the high numerical cost of the calculations as compared to PWD ones. The next step of our work will involve inclusion of the full $3 \mathrm{~N}$ Faddeev equation in the calculation and optimizing the code to make it numerically more effective. We believe that this can be achieved by introducing a general form of the $3 \mathrm{~N}$ scattering operator. This form would be constructed to obey the parity, time reversal and rotation symmetries implicitly.

The project was financed from the resources of the National Science Center (Poland) under grants No. DEC2013/11/N/ST2/03733 and DEC-2013/10/M/ST2/00420. Some numerical calculations have been performed on the supercomputer clusters of the JSC, Jülich, Germany. This work was additionally supported by U.S. NSF Grant No. 1415656 and the U.S. DOE grant No. DE-FG0208ER41533. 

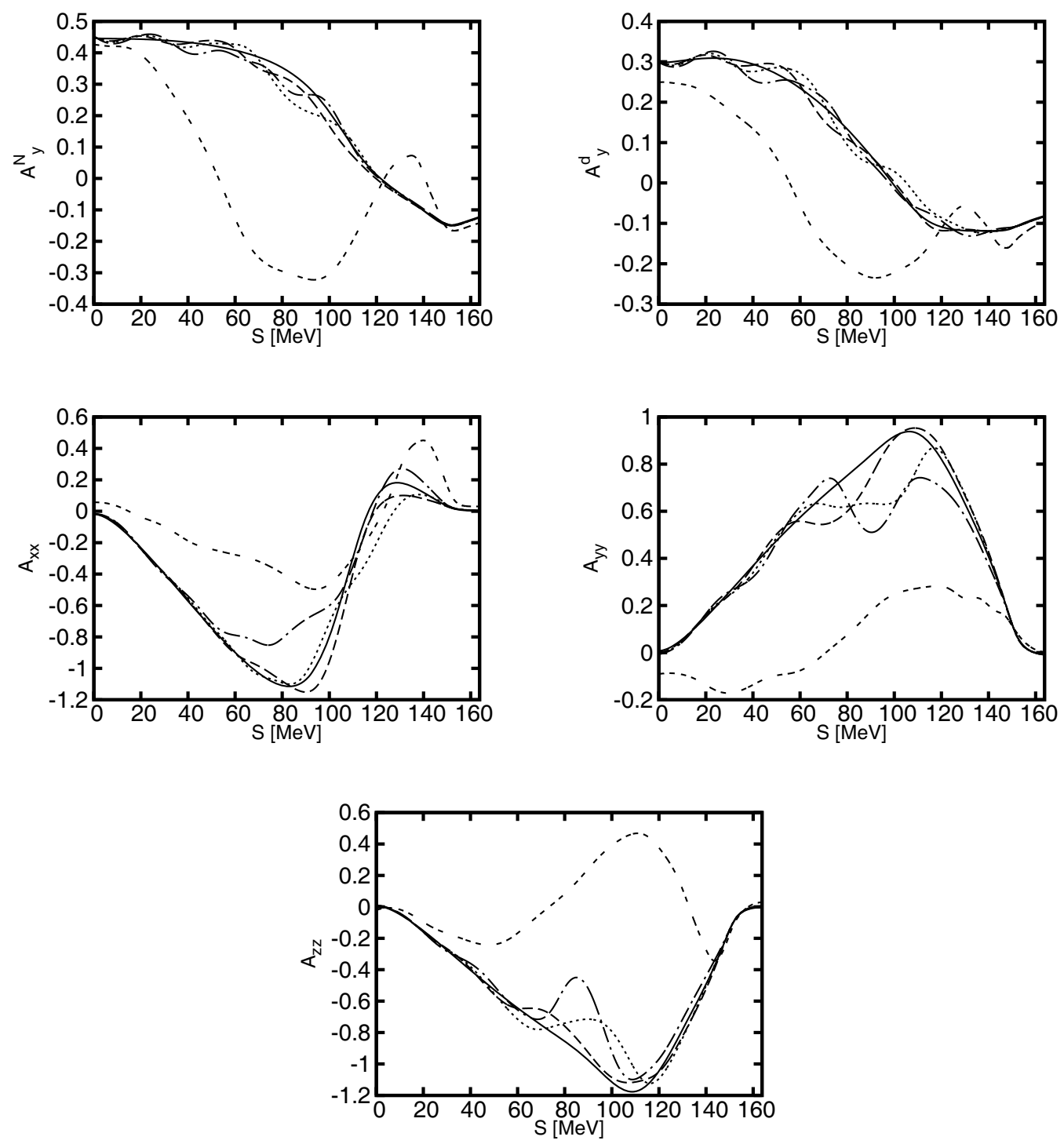

Fig. 6. The same as in fig. 4 but for $190 \mathrm{MeV}$. The solid line is calculated using the 3D formalism. The dash-dotted, dotted and dashed lines are calculated using PWD with $J_{\max }=\frac{21}{2}, \frac{23}{2}, \frac{25}{2}$ and $j_{\max }=8$. Convergence is observed, however, for some kinematical regimes, a higher number of partial waves should be included in order to get full agreement. This was difficult due to the high memory requirements of the PWD code. PWD results that take into account the full equation for $\breve{T}(5)$ are plotted using the double-dashed line for comparison, they are obtained using $J_{\max }=\frac{25}{2}$ and $j_{\max }=7$. 

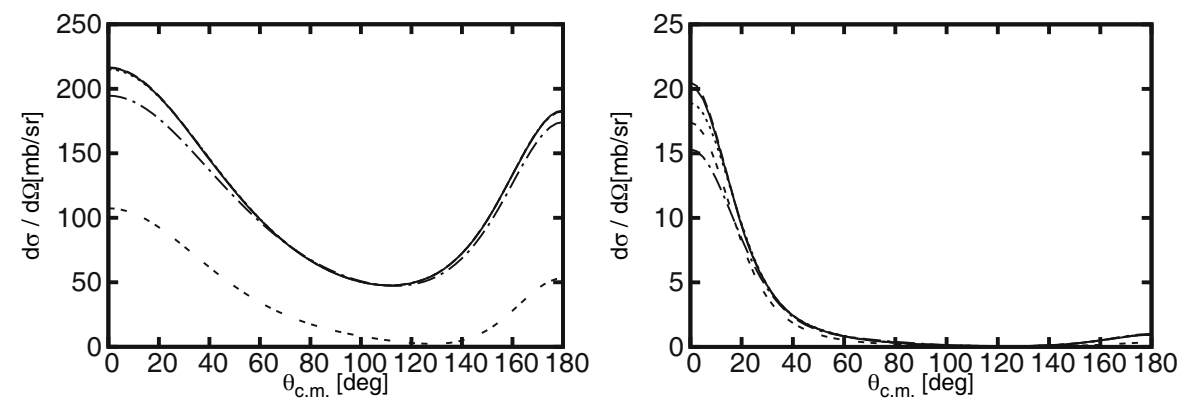

Fig. 7. Left panel: The differential cross-section for $25 \mathrm{MeV}$ neutron-deuteron elastic scattering in the c.m. frame as a function of the center-of-mass angle $\theta_{\text {c.m. }}$. Right panel: the same but for $190 \mathrm{MeV}$. The solid line is obtained from 3D calculations. The partial wave calculations (plotted using dash-dotted, dotted and dashed lines) are converging to the 3D results (solid line) for large angular momenta of the $2-3$ subsystem $j_{\max }$ used in the calculations $\left(j_{\max }=1,3,5\right.$ for $25 \mathrm{MeV}$ and $j_{\max }=3,5,8$ for $190 \mathrm{MeV}$, respectively). The total angular momentum used in the PWD calculations is $J_{\max }=\frac{25}{2}$. PWD results that take into account the full equation for $\check{T}(5)$ are plotted using the double-dashed line for comparison, they are obtained using $J_{\text {max }}=\frac{25}{2}$ and $j_{\max }=7$.
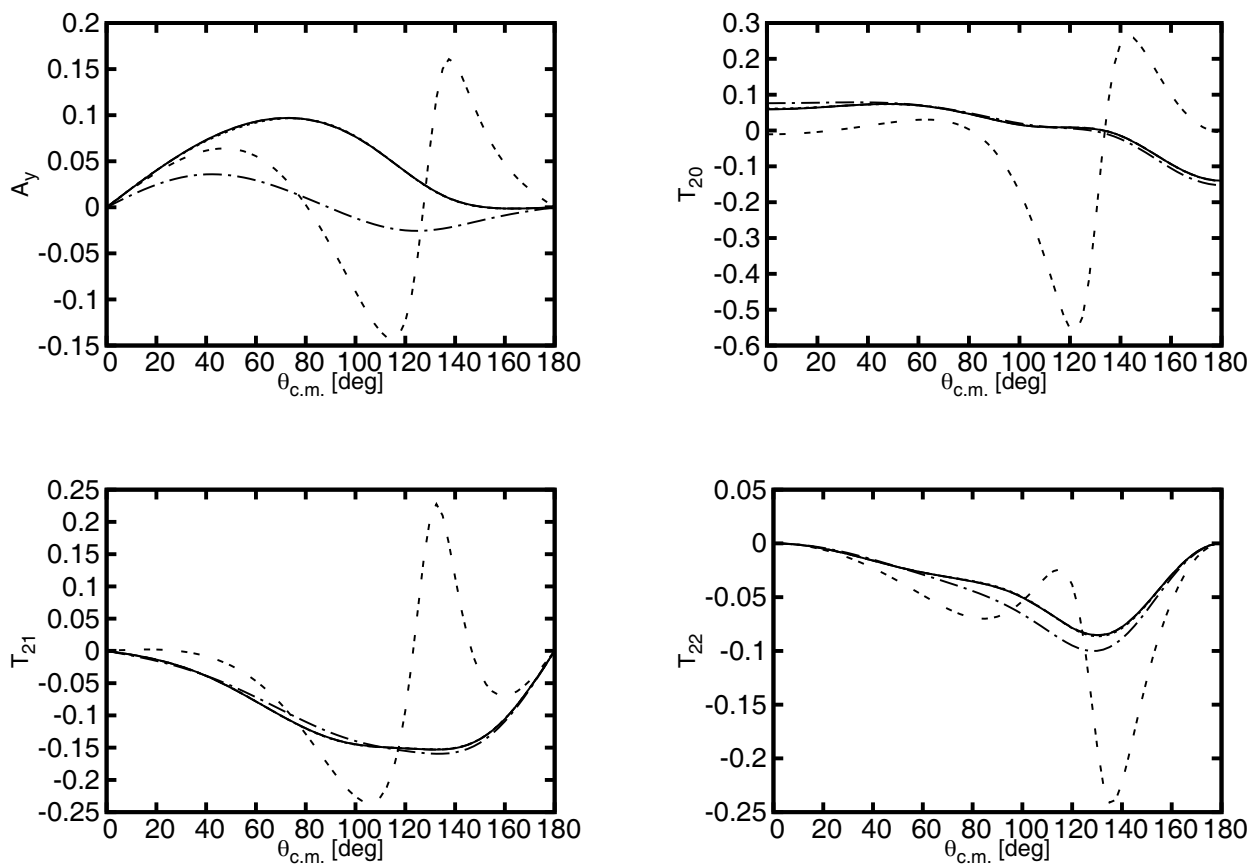

Fig. 8. The spin observables for elastic $25 \mathrm{MeV}$ neutron-deuteron scattering in the c.m. frame as a function of the center-of-mass scattering angle $\theta_{\text {c.m. }} . T_{20}, T_{21}, T_{22}$ are the deuteron analyzing powers and $A_{y}$ is the nucleon analyzing power [1]. The solid line is obtained from 3D calculations. The partial wave results, plotted using dash-dotted, dotted and dashed lines, are for angular momenta of the 2-3 subsystem $j_{\max }=1,3$ and 5 , respectively. The total angular momentum used in the PWD calculations is $J_{\max }=\frac{25}{2}$. PWD results that take into account the full equation for $\check{T}(5)$ are plotted using the double-dashed line for comparison, they are obtained using $J_{\max }=\frac{25}{2}$ and $j_{\max }=7$. 

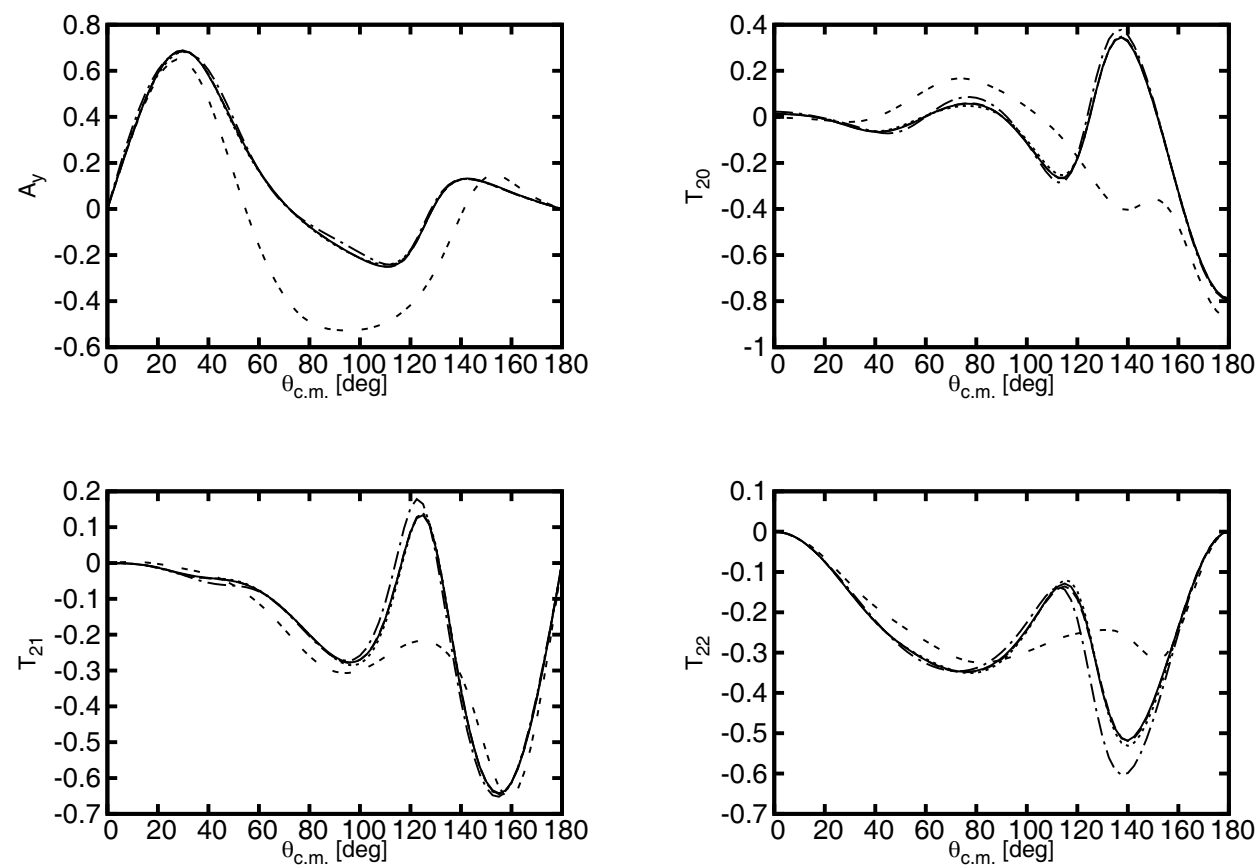

Fig. 9. Same as in fig. 8 but for $190 \mathrm{MeV}$ and $j_{\max }=3,5,8$ plotted using dash-dotted, dotted and dashed lines, respectively. The 3D results plotted using a solid line merge with the dashed, $j_{\max }=8$, result. PWD results that take into account the full $\check{T}$ (see eq. (5)) are plotted using the double-dashed line for comparison, they are obtained using $J_{\max }=\frac{25}{2}$ and $j_{\max }=7$.
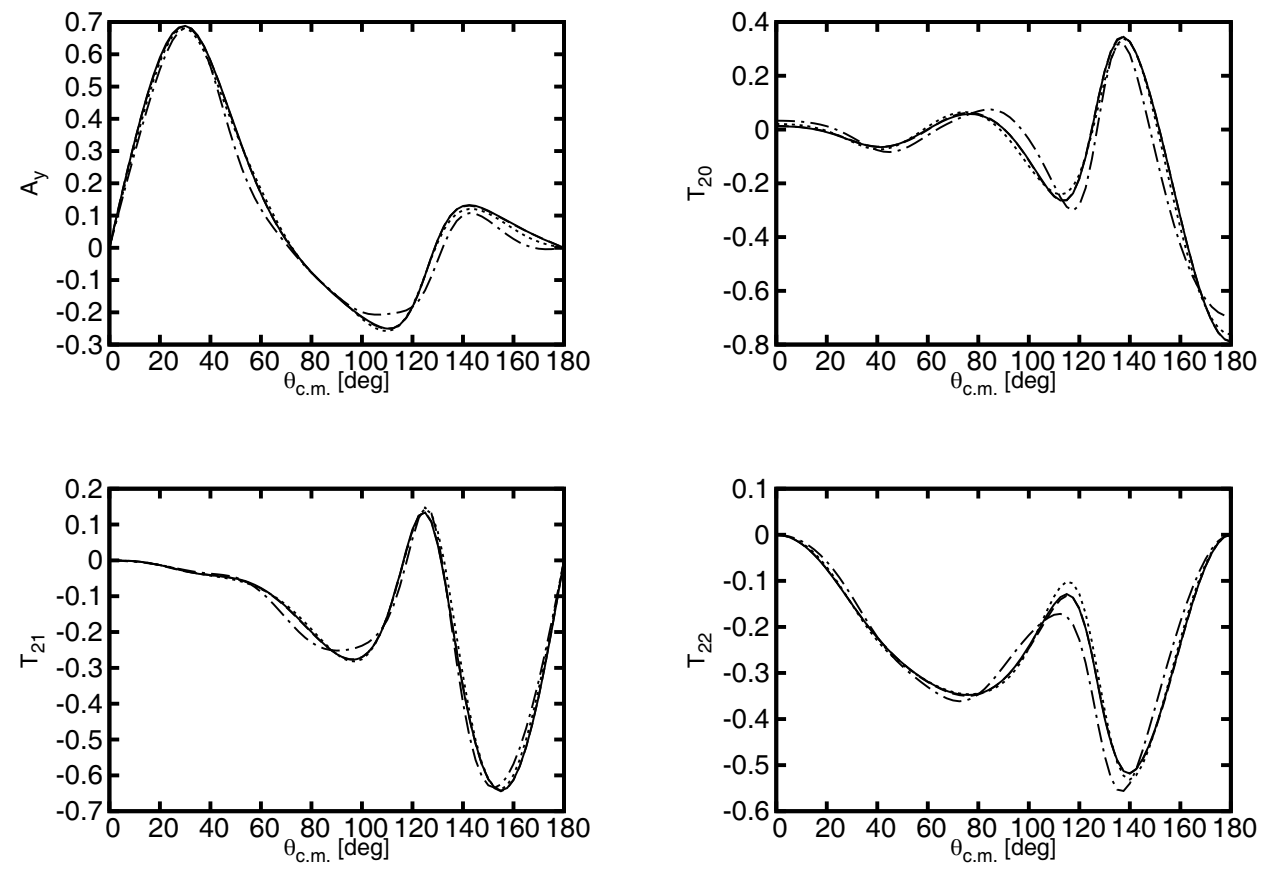

Fig. 10. Same as in fig. 9 but for $190 \mathrm{MeV}$ and the partial waves with $j_{\max }=7$ and total angular momenta $J_{\max }=\frac{11}{2}, \frac{15}{2}, \frac{25}{2}$ plotted using dash-dotted, dotted and dashed lines, respectively. The $3 \mathrm{D}$ results plotted using the solid line merge with the dashed, $J_{\max }=\frac{25}{2}$, result. 


\section{Appendix A. Breakup operators and momenta}

Table 1. The permutation operators in the breakup process, see eq. (23).

\begin{tabular}{|c|cc|}
\hline$c$ & $\check{P}_{c}^{\mathrm{BL}}$ & $\check{P}_{c}^{\mathrm{BR}}$ \\
\hline 1 & $\check{1}$ & $\check{P}_{12} \check{P}_{23}$ \\
2 & $\check{1}$ & $\check{P}_{13} \check{P}_{23}$ \\
3 & $\check{P}_{12} \check{P}_{23}$ & $\check{P}_{12} \check{P}_{23}$ \\
4 & $\check{P}_{12} \check{P}_{23}$ & $\check{P}_{13} \check{P}_{23}$ \\
5 & $\check{P}_{13} \check{P}_{23}$ & $\check{P}_{12} \check{P}_{23}$ \\
6 & $\check{P}_{13} \check{P}_{23}$ & $\check{P}_{13} \check{P}_{23}$ \\
\hline
\end{tabular}

Table 2. The momenta used in the description of the breakup process, see eq. (23).

\begin{tabular}{|c|cc|}
\hline$c$ & $\boldsymbol{p}_{c}^{\mathrm{BL}}$ & $\boldsymbol{p}_{c}^{\mathrm{BR}}$ \\
\hline 1 & $\boldsymbol{p}^{f}$ & $\boldsymbol{q}_{i}+\frac{\boldsymbol{q}^{f}}{2}$ \\
2 & $\boldsymbol{p}^{f}$ & $-\boldsymbol{q}_{i}-\frac{\boldsymbol{q}^{f}}{2}$ \\
3 & $\frac{1}{4}\left(-2 \boldsymbol{p}^{f}-3 \boldsymbol{q}^{f}\right)$ & $\boldsymbol{q}_{i}+\frac{\boldsymbol{p}^{f}}{2}-\frac{\boldsymbol{q}^{f}}{4}$ \\
4 & $\frac{1}{4}\left(-2 \boldsymbol{p}^{f}-3 \boldsymbol{q}^{f}\right)$ & $\frac{1}{4}\left(-4 \boldsymbol{q}_{i}-2 \boldsymbol{p}^{f}+\boldsymbol{q}^{f}\right)$ \\
5 & $\frac{1}{4}\left(3 \boldsymbol{q}^{f}-2 \boldsymbol{p}^{f}\right)$ & $\boldsymbol{q}_{i}-\frac{\boldsymbol{p}^{f}}{2}-\frac{\boldsymbol{q}^{f}}{4}$ \\
6 & $\frac{1}{4}\left(3 \boldsymbol{q}^{f}-2 \boldsymbol{p}^{f}\right)$ & $\frac{1}{4}\left(-4 \boldsymbol{q}_{i}+2 \boldsymbol{p}^{f}+\boldsymbol{q}^{f}\right)$ \\
\hline \hline$c$ & $\boldsymbol{q}_{c}^{\mathrm{BQ}}$ & $\boldsymbol{p}_{c}^{\mathrm{BD}}$ \\
\hline 1 & $\boldsymbol{q}^{f}$ & $-\frac{\boldsymbol{q}_{i}}{2}-\boldsymbol{q}^{f}$ \\
2 & $\boldsymbol{q}^{f}$ & $\frac{\boldsymbol{q}_{i}}{2}+\boldsymbol{q}^{f}$ \\
3 & $\boldsymbol{p}^{f}-\frac{\boldsymbol{q}^{f}}{2}$ & $\frac{1}{2}\left(-\boldsymbol{q}_{i}-2 \boldsymbol{p}^{f}+\boldsymbol{q}^{f}\right)$ \\
4 & $\boldsymbol{p}^{f}-\frac{\boldsymbol{q}^{f}}{2}$ & $\frac{1}{2}\left(\boldsymbol{q}_{i}+2 \boldsymbol{p}^{f}-\boldsymbol{q}^{f}\right)$ \\
5 & $-\boldsymbol{p}^{f}-\frac{\boldsymbol{q}^{f}}{2}$ & $\frac{1}{2}\left(-\boldsymbol{q}_{i}+2 \boldsymbol{p}^{f}+\boldsymbol{q}^{f}\right)$ \\
6 & $-\boldsymbol{p}^{f}-\frac{\boldsymbol{q}^{f}}{2}$ & $\frac{1}{2}\left(\boldsymbol{q}_{i}-2 \boldsymbol{p}^{f}-\boldsymbol{q}^{f}\right)$ \\
\hline
\end{tabular}

Table 3. The permutation operators in the $\check{P} G_{0}^{-1}$ part of the elastic amplitude, see eq. (26).

\begin{tabular}{|c|c|}
\hline$c$ & $\check{P}_{c}^{\mathrm{E}}$ \\
\hline 1 & $\check{P}_{12} \check{P}_{23}$ \\
2 & $\check{P}_{13} \check{P}_{23}$ \\
\hline
\end{tabular}

Table 4. The momenta in the $\check{P} G_{0}^{-1}$ part of the elastic amplitude, see eq. (26).

\begin{tabular}{|c|cc|}
\hline$c$ & $\boldsymbol{p}_{c}^{\mathrm{EL}}$ & $\boldsymbol{p}_{c}^{\mathrm{ER}}$ \\
\hline 1 & $-\boldsymbol{q}_{f}-\frac{\boldsymbol{q}_{i}}{2}$ & $\frac{\boldsymbol{q}_{f}}{2}+\boldsymbol{q}_{i}$ \\
2 & $\boldsymbol{q}_{f}+\frac{\boldsymbol{q}_{i}}{2}$ & $-\frac{\boldsymbol{q}_{f}}{2}-\boldsymbol{q}_{i}$ \\
\hline
\end{tabular}

Table 5. The permutation operators in the $\check{P} \check{t} \check{P}$ part of the elastic amplitude, see eq. (27).

\begin{tabular}{|c|cc|}
\hline$c$ & $\check{P}_{c}^{\mathrm{EL}}$ & $\check{P}_{c}^{\mathrm{ER}}$ \\
\hline 1 & $\check{P}_{12} \check{P}_{23}$ & $\check{P}_{12} \check{P}_{23}$ \\
2 & $\check{P}_{12} \check{P}_{23}$ & $\check{P}_{13} \check{P}_{23}$ \\
3 & $\check{P}_{13} \check{P}_{23}$ & $\check{P}_{12} \check{P}_{23}$ \\
4 & $\check{P}_{13} \check{P}_{23}$ & $\check{P}_{13} \check{P}_{23}$ \\
\hline
\end{tabular}

Table 6. The momenta in the $\check{P} \breve{t} \breve{P}$ part of the elastic amplitude, see eq. (27).

\begin{tabular}{|c|cccc|}
\hline$c$ & $\boldsymbol{p}_{c}^{\mathrm{EDL}}$ & $\boldsymbol{p}_{c}^{\mathrm{ETL}}$ & $\boldsymbol{p}_{c}^{\mathrm{ETR}}$ & $\boldsymbol{p}_{c}^{\mathrm{EDR}}$ \\
\hline 1 & $\frac{\boldsymbol{q}^{f}}{2}+\boldsymbol{q}$ & $-\boldsymbol{q}^{f}-\frac{\boldsymbol{q}}{2}$ & $\boldsymbol{q}_{i}+\frac{\boldsymbol{q}}{2}$ & $-\frac{\boldsymbol{q}_{i}}{2}-\boldsymbol{q}$ \\
2 & $\frac{\boldsymbol{q}^{f}}{2}+\boldsymbol{q}$ & $-\boldsymbol{q}^{f}-\frac{\boldsymbol{q}}{2}$ & $-\boldsymbol{q}_{i}-\frac{\boldsymbol{q}}{2}$ & $\frac{\boldsymbol{q}_{i}}{2}+\boldsymbol{q}$ \\
3 & $-\frac{\boldsymbol{q}^{f}}{2}-\boldsymbol{q}$ & $\boldsymbol{q}^{f}+\frac{\boldsymbol{q}}{2}$ & $\boldsymbol{q}_{i}+\frac{\boldsymbol{q}}{2}$ & $-\frac{\boldsymbol{q}_{i}}{2}-\boldsymbol{q}$ \\
4 & $-\frac{\boldsymbol{q}^{f}}{2}-\boldsymbol{q}$ & $\boldsymbol{q}^{f}+\frac{\boldsymbol{q}}{2}$ & $-\boldsymbol{q}_{i}-\frac{\boldsymbol{q}}{2}$ & $\frac{\boldsymbol{q}_{i}}{2}+\boldsymbol{q}$ \\
\hline
\end{tabular}

\section{Appendix B. Explicit forms of the permutation operators in $3 \mathrm{~N}$ spin (isospin) space}

The following matrix representations of $3 \mathrm{~N}$ spin (isospin) permutation operators use the single particle basis from table 7:

$$
\left[P_{12}\right]^{3 \mathrm{~N} \text { spin (isospin) }}=\left(\begin{array}{llllllll}
1 & 0 & 0 & 0 & 0 & 0 & 0 & 0 \\
0 & 1 & 0 & 0 & 0 & 0 & 0 & 0 \\
0 & 0 & 0 & 0 & 1 & 0 & 0 & 0 \\
0 & 0 & 0 & 0 & 0 & 1 & 0 & 0 \\
0 & 0 & 1 & 0 & 0 & 0 & 0 & 0 \\
0 & 0 & 0 & 1 & 0 & 0 & 0 & 0 \\
0 & 0 & 0 & 0 & 0 & 0 & 1 & 0 \\
0 & 0 & 0 & 0 & 0 & 0 & 0 & 1
\end{array}\right),
$$


Table 7. Reference list of $3 \mathrm{~N}$ spin(isospin) basis states $\left|\frac{1}{2}, \nu_{1}^{\text {spin(isospin) }}(i)\right\rangle^{\operatorname{spin}(\text { isospin })} \otimes\left|\frac{1}{2}, \nu_{2}^{\text {spin(isospin) }}(i)\right\rangle^{\operatorname{spin}(\text { isospin })} \otimes$ $\left|\frac{1}{2}, \nu_{3}^{\text {spin(isospin) }}(i)\right\rangle^{\operatorname{spin}(\text { isospin) }}$ quantum numbers. $i$ is the number of the basis state and $\nu_{j}^{\operatorname{spin}(\text { isospin) }}(i)$ is the projection of the spin (isospin) of particle $i$.

\begin{tabular}{|c|ccc|}
\hline$i$ & $\nu_{1}^{\text {(iso)spin }}(i)$ & $\nu_{2}^{(\text {iso }) \operatorname{spin}}(i)$ & $\nu_{3}^{\text {(iso)spin }}(i)$ \\
\hline 1 & $-\frac{1}{2}$ & $-\frac{1}{2}$ & $-\frac{1}{2}$ \\
2 & $-\frac{1}{2}$ & $-\frac{1}{2}$ & $\frac{1}{2}$ \\
3 & $-\frac{1}{2}$ & $\frac{1}{2}$ & $-\frac{1}{2}$ \\
4 & $-\frac{1}{2}$ & $\frac{1}{2}$ & $\frac{1}{2}$ \\
5 & $\frac{1}{2}$ & $-\frac{1}{2}$ & $-\frac{1}{2}$ \\
6 & $\frac{1}{2}$ & $-\frac{1}{2}$ & $\frac{1}{2}$ \\
7 & $\frac{1}{2}$ & $\frac{1}{2}$ & $-\frac{1}{2}$ \\
8 & $\frac{1}{2}$ & $\frac{1}{2}$ & $\frac{1}{2}$ \\
\hline
\end{tabular}

Open Access This is an open access article distributed under the terms of the Creative Commons Attribution License (http://creativecommons.org/licenses/by/4.0), which permits unrestricted use, distribution, and reproduction in any medium, provided the original work is properly cited.

\section{References}

1. W. Glöckle, H. Witała, D. Hüber, H. Kamada, J. Golak, Phys. Rep. 274, 107 (1996).

2. H. Witała, W. Glöckle, J. Golak, H. Kamada, J. KurośŻołnierczuk, A. Nogga, R. Skibiński, Phys. Rev. C 63, 024007 (2001).

3. A. Deltuva, A.C. Fonseca, A. Kievsky, S. Rosati, P.U. Sauer, M. Viviani, Phys. Rev. C 71, 064003 (2005).

4. M.N. Platonova, V.I. Kukulin, Phys. At. Nucl. 73, 86 (2010), Yad. Fiz. 73, 90 (2010).

5. R. Machleidt, Advances in Nuclear Physics, Vol. 19, The Meson Theory of Nuclear Forces and Nuclear Structure (Springer, US, 1989).
6. R.B. Wiringa, V.G.J. Stoks, R. Schiavilla, Phys. Rev. C 51, 38 (1995).

7. R. Machleidt, F. Sammarruca, Y. Song, Phys. Rev. C 53, R1483 (1996).

8. V.G.J. Stoks, R.A.M. Klomp, C.P.F. Terheggen, J.J. de Swart, Phys. Rev. C 49, 2950 (1994).

9. D.R. Entem, R. Mechleidt, Phys. Rev. C 68, 041001 (2003).

10. E. Epelbaum, W. Glöckle, Ulf-G. Meißner, Nucl. Phys. A 747, 362 (2005).

11. H. Witała, J. Golak, R. Skibiński, K. Topolnicki, J. Phys. G: Nucl. Part. Phys. 41, 094001 (2014).

12. H. Witała, W. Glöckle, D. Hüber, J. Golak, H. Kamada, Phys. Rev. Lett. 81, 1183 (1998).

13. K. Sekiguchi et al., Phys. Rev. C 89, 064007 (2014).

14. E. Stephan et al., Eur. Phys. J. A 49, 36 (2013).

15. I. Ciepał et al., Phys. Rev. C 85, 017001 (2012).

16. H. Witała, J. Golak, R. Skibiński, W. Glöckle, H. Kamada, W.N. Polyzou, Phys. Rev. C 83, 044001 (2011).

17. H. Witała, J. Golak, R. Skibiński, W. Glöckle, H. Kamada, W.N. Polyzou, Phys. Rev. C 88, 069904 (2013).

18. W. Glöckle, The Quantum Mechanical Few-Body Problem (Springer-Verlag, Berlin-Heidelberg, 1983).

19. W. Glöckle, I. Fachruddin, Ch. Elster, J. Golak, R. Skibiński, H. Witała, Eur. Phys. J. A 43, 339 (2010).

20. J. Golak, W. Glöckle, R. Skibiński, H. Witała, D. Rozpȩdzik, K. Topolnicki, I. Fachruddin, Ch. Elster, A. Nogga, Phys. Rev. C 81, 034006 (2010).

21. J. Golak, R. Skibiński, H. Witała, K. Topolnicki, W. Glöckle, A. Nogga, H. Kamada, Few-Body Syst. 53, 237 (2012).

22. K. Topolnicki, PhD thesis, Jagiellonian University (2014) unpublished, available online http://www.fais. uj.edu.pl/dla-studentow/studia-doktoranckie/ prace-doktorskie\#2014.

23. Wolfram Research Inc. Mathematica Version 10.0 (2014) Wolfram Research Inc. Champaign, Illinois.

24. I. Fachruddin, Ch. Elster, W. Glöckle, Phys. Rev. C 63, 054003 (2001).

25. L. Wolfenstein, Phys. Rev. 96, 1654 (1954).

26. Y. Saad, Iterative methods for sparse linear systems (SIAM Philadelphia, PA, USA, 2003). 